\title{
Superhydrophobic Cerium-Based Coatings on Al-Mg Alloys and Aluminized Steel
}

\author{
Guillermo Guerrero-Vaca ${ }^{1}{ }^{\circledR}$, Miguel A. Rodríguez-Valverde ${ }^{2}{ }^{\circledR}$, Pedro Castilla-Montilla ${ }^{1}$, \\ Francisco Alguacil-Salamanca ${ }^{1}$, Óscar Rodríguez-Alabanda ${ }^{1, *}$ (D) Pablo E. Romero ${ }^{1}[$, \\ Esther Molero-Romero ${ }^{1}$ and F. Javier Montes Ruiz-Cabello ${ }^{2}$ \\ 1 Department of Mechanical Engineering, University of Cordoba, Medina Azahara Avenue, 514071 Cordoba, \\ Spain; guillermo.guerrero@uco.es (G.G.-V.); pecasmon@hotmail.com (P.C.-M.); fjalguacil@live.com (F.A.-S.); \\ p62rocap@uco.es (P.E.R.); esther.molero@uco.es (E.M.-R.) \\ 2 Laboratoy of Surface and Interface Physics (LSIP), Applied Physics Department, Faculty of Sciences, \\ University of Granada, Campus de Fuentenueva s/n, ES 18071 Granada, Spain; marodri@ugr.es (M.A.R.-V.); \\ fjmontes@ugr.es (F.J.M.R.-C.) \\ * Correspondence: osrodala@uco.es; Tel.: +34-957-212-235
}

Received: 11 October 2019; Accepted: 17 November 2019; Published: 20 November 2019

check for updates

\begin{abstract}
Aluminum-magnesium (Al-Mg) alloy and aluminum-coated steel (aluminized steel) are typically used for the manufacturing of baking trays and molds. For these applications, these materials must be modified to develop release and hydrophobic properties. With this aim, the bare substrates are typically coated with low-surface energy materials such as fluoropolymers, elastomers, or sol-gel layers. In this work, some alternative strategies to prepare these functional surfaces are presented. We used three-step processes involving (i) micro-texturing, (ii) nano layer deposition through immersion and electrodeposition, and (iii) hydrophobization. The raw substrates were sanded or sandblasted at the micro scale, accordingly. Texturization at the nano scale was achieved with a cerium layer formed by electrodeposition or solution immersion. The cerium layers were hydrophobized with fatty acids. The wetting properties of the samples were studied with tilting-plate and bouncing drop methods. We measured the surface roughness of the samples by contact profiling and analyzed their surface morphology using a field emission scanning electron microscope (FESEM). The elemental chemical composition of the samples was analyzed by energy-dispersive X-ray spectroscopy (EDX). The wettability results indicated that the best performance for the $\mathrm{Al}-\mathrm{Mg}$ substrates was reached by sandblasting and later immersion in a cerium nitrate solution. For aluminized steel substrates, the best results were obtained with both electrodeposition and immersion methods using a cerium chloride solution.
\end{abstract}

Keywords: aluminized steel; aluminium-magnesium alloy; superhydrophobic surfaces; sandblasting; cerium coating; electrodeposition; immersion

\section{Introduction}

Current interest in hydrophobic and low-adhesion surfaces has grown and numeorus preparation methods have been developed. Many substrates of industrial interest are modified to reduce their adhesion with liquids/solids. Release/demolding coatings are typically prepared for food, doughs, liquids, gels, and foams. Aluminium-magnesium (Al-Mg) alloy and aluminized steel (AS) substrates are used in industry to produce trays, utensils, and molds for baking ovens [1]. However, these metallic materials are sticky and hydrophilic. The incorporation of coatings to decrease their intrinsic high wettability and adhesion is mandatory. So far, the most extended engineering solutions are the use of polymer-based or ceramic coatings [2-4]. Although the efficiency of the current solutions is fine 
enough, it is challenging to obtain surfaces with enhanced non-stick properties. The current coatings used in industry are resistant, but their low-adhesion properties are not optimal yet.

Tailoring the wetting properties of solid surfaces is desirable in many fields. The limit for the lowest adhesion properties is found with so-called liquid-repellent surfaces [5]. These surfaces may incorporate further applications such as anti-icing [6], self-cleaning [7], anti-corrosion [8], drug reduction [9], anti-fouling and anti-biofouling [10], microfluidic device construction [11], anti-reflective [12], and antibacterial effects [13]. In the literature, one may find a wide variety of methods to obtain superhydrophobic surfaces, including silanization by liquid or chemical vapor deposition (CVD) [14], sol-gel coatings [15], chemical immersion [16], production of roughness on inherent hydrophobic polymer layers [17], nanoparticle coatings [18], micro-machining [19], layer-by-layer deposition [20], electrodeposition [21], lithography [22], chemical etching [23], and silicone coatings [24].

Superhydrophobicity is qualitatively explained through the Cassie-Baxter equation, i.e.,

$$
\cos \theta_{E q}=f r_{w} \cos \theta_{i}-(1-f)
$$

where $\theta_{E q}$ is the drop contact agle, $f$ is the ratio between the wet area and the projected (apparent) contact area, $r_{w}$ is the roughness factor of that wet area, and $\theta_{i}$ is the intrinsic contact angle of the material. From Equation (1), the most relevant parameter is $f$, because the lower the real contact between the drop and the solid surface, the higher the contact angle and the drop mobility. Many different studies report that the minimization of $f$ is reached for intrinsically hydrophobic surfaces with a hierarchical structured surface. A distribution of micro- and nano-scale surface features is responsible for the highest water repellency. Most of the experimental strategies used in the literature to produce superhydrophobic surfaces are based on the minimization of the $f$ factor through scanning the role of certain parameters or settings used during the fabrication process.

However, the experimental acquisition of the $f$ parameter in Equation (1) is not reported in most cases because it would require a microscopic observation of the entire contact area by using sophisticated techniques and the estimation of the equilibrium and intrinsic contact angles (hardly measurable quantities). It is well accepted that the minimization of the $f$ parameter is assumed to be reached indirectly when the highest drop contact angle and the lowest sliding angle are observed.

The preparation of superhydrophobic surfaces on metal substrates is particularly useful from an industrial point of view. A frequent strategy for producing durable superhydrophobic metallic materials is the creation of a micro-/nano-scale surface structure followed by hydrophobization. Due to their physical robustness and chemical inertia, the deposition of ceria coatings $[25,26]$ on metal surfaces is a promising route. Cerium is the most abundant lanthanide. It is a good heat and electricity conductor and is widely used in polishing materials, automobile exhaust catalysts, gas sensors, and electrolytic materials. Moreover, cerium can be used for agricultural and food contact applications, with precautions [27].

In this work, we propose top-down and bottom-up strategies to produce superhydrophobic cerium-based coatings on Al-Mg alloy and AS substrates. Similar strategies have been used earlier for different substrates [8,28,29]. Unlike other works reported for the same materials and similar purposes, the protocols described here do not require etching with strong acids for the texturization [23]. Our procedure aimed to create mechanically a specific micro-texture, to introduce durable nano-asperities with cerium layers deposited on the rough substrate, and to hydrophobize the uppermost layer. The substrates were polished with abrasive papers or sandblasted, accordingly. The second step was cerium deposition conducted by electrodeposition or immersion (surface growth by "soft" self-assembly), using cerium chloride or cerium nitrate, respectively. The hydrophobization was conducted during the electrodeposition (myristic acid) or after immersion (stearic acid). We identified water-repellent Al-Mg alloys and AS substrates and found the least aggressive and most efficient texturing process. 


\section{Materials and Methods}

\subsection{Surface Preparation}

Several sheets of Al-Mg alloy (EN AW 5754 H32) and AS (DX51D AS120 B) were supplied by CAMEBE (Carpinteria Metalica Bengolea, Castro Urdiales, Spain). The thickness of the Al-Mg sheets, as provided by the manufacturer, was $1.2 \mathrm{~mm}$, and the thickness was $1 \mathrm{~mm}$ for the AS sheets. The sheets were cut to prepare 48 samples $(17 \mathrm{~mm} \times 38 \mathrm{~mm})$ of each material.

The elemental chemical composition of the as-received substrates was analyzed by energy-dispersive X-ray microanalysis (EDX) with a JEOL JSM 6300 SEM (Jeol USA, Peabody, MA, USA). The results for the chemical composition of each material are shown in Table 1.

Table 1. Elemental chemical composition (\% in weight) of the Al-Mg alloy and aluminized steel (AS) samples used in this study.

\begin{tabular}{cccccccccc}
\hline Material & $\mathbf{M g}$ & $\mathbf{S i}$ & $\mathbf{F e}$ & $\mathbf{M n}$ & $\mathbf{C u}$ & $\mathbf{Z n}$ & $\mathbf{C r}$ & $\mathbf{T i}$ & $\mathbf{A l}$ \\
\hline Al-Mg alloy & 2.5 & 0.46 & 0.56 & 0.27 & - & - & - & - & Rest \\
AS & 0.01 & 8.3 & 0.45 & 0.1 & 0.05 & - & - & - & Rest \\
\hline
\end{tabular}

\subsubsection{Cathodic Electrodeposition of Cerium-Based Coatings}

We selected 24 samples of the Al-Mg alloy and 24 samples of AS for the electrodeposition. All the surfaces were polished manually with \#2500 sandpaper to increase their micro-roughness and to remove impurities or organic traces. This texturing process, as explained below, was enough to produce superhydrophobic surfaces on the Al-Mg samples. However, it was unable to induce superhydrophobicity on the AS samples. For this reason, a subsequent sandblasting was carried out on the AS samples. We used a Sand Blast Cabinet CAT-990 (Aslak S.L, San Quirze del Valles, Barcelona, Spain) for this purpose. We explored different pressures $(0.2,0.3,0.4$, and $0.5 \mathrm{MPa})$ and two blasting times (5 and $10 \mathrm{~s}$ ). We used the brown corundum RBT9 Gr.60 (MPA, Cornellá, Barcelona, Spain). Higher pressures or longer blasting times were avoided to minimize damage of the AS samples [1]. The textured samples were carefully cleaned by sonication in acetone for 5 min and, afterwards, in ethanol for $5 \mathrm{~min}$.

The clean textured samples were dried at room temperature and then nano-textured and hydrophobized simultaneously by the electrodeposition method. All the treatments were performed at room temperature. We used a piece of platinum as the anode and the Al-Mg or AS samples as the cathode, accordingly. These samples were fixed inside a flask with a separation distance of $25 \mathrm{~mm}$. The flask was then filled with an electrolyte solution under constant agitation by means of a magnetic stirrer. The electrolyte solution was a mixture of $100 \mathrm{~mL}$ ethanol, $100 \mathrm{~mL}$ cerium (III) chloride hepta-hydrate $\left(\mathrm{CeCl}_{3}-7 \mathrm{H}_{2} \mathrm{O}\right) 0.10 \mathrm{~mol} \cdot \mathrm{L}^{-1}$ and $100 \mathrm{~mL}$ myristic acid $\left(\mathrm{CH}_{3}\left(\mathrm{CH}_{2}\right)_{12} \mathrm{COOH}\right)$ 0.30 mol. $\mathrm{L}^{-1}$ [25]. All the reagents were supplied by Sigma-Aldrich. Both the cathode and the anode were connected to a DC power supplier (Figure 1) at different voltages of 10, 20, 30, and $40 \mathrm{~V}$ for intervals of $20 \mathrm{~min} / 30 \mathrm{~min}$ for the Al-Mg alloys and $10 \mathrm{~min} / 20 \mathrm{~min}$ for the AS samples. Under these conditions, the current density passing through the samples varied from 0.01 to $0.03 \mathrm{~mA} / \mathrm{cm}^{2}$. Finally, the samples were rinsed out with deionized water and then dried under a flow of hot air. 


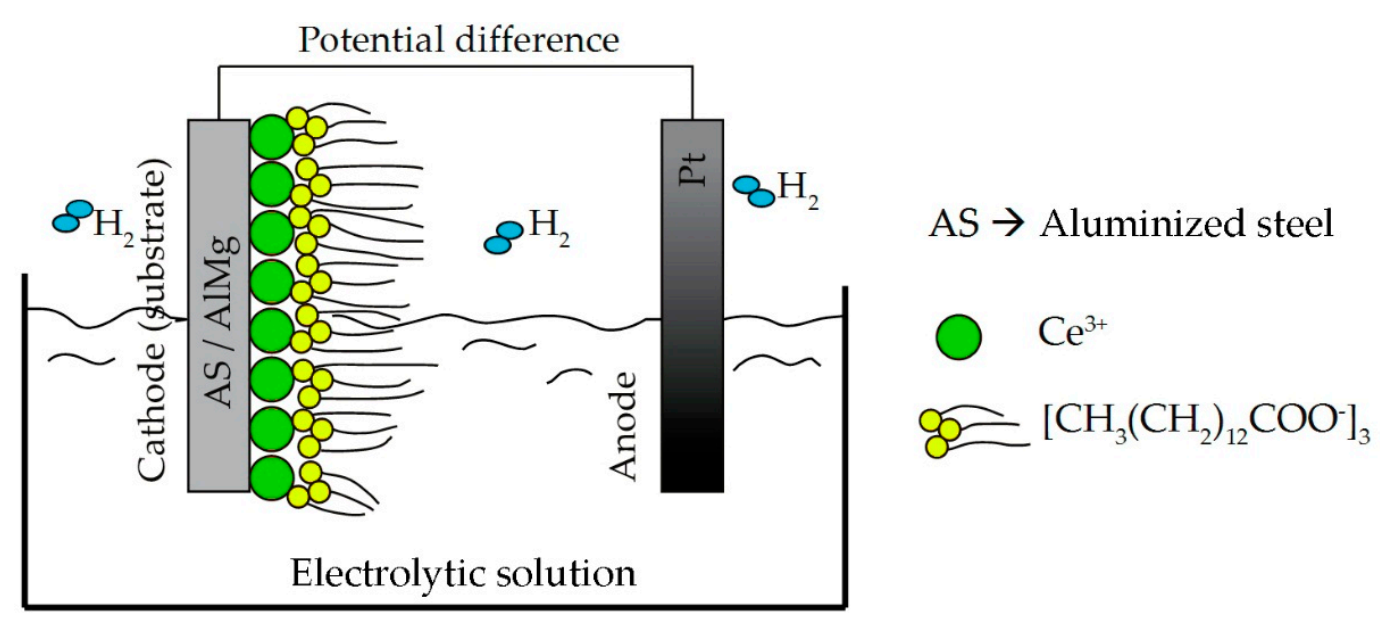

Figure 1. Diagram for the electrodeposition of cerium-based coatings on Al-Mg and AS samples.

\subsubsection{Deposition of Cerium-Based Coatings by Immersion}

We used 12 samples of each substrate for deposition by immersion. Unlike the electrodeposition, a previous sandblasting of the Al-Mg alloy samples was required. This way, a combination of manual polishing (with \#2500 sandpaper) and subsequent sandblasting was used for the samples. The polished samples were sandblasted with a pressure range of $0.2,0.4$, and $0.6 \mathrm{MPa}$ for 10 and $15 \mathrm{~s}$ for the Al-Mg alloys. For the AS samples, we used a pressure range of $0.2,0.3,0.4$, and $0.5 \mathrm{MPa}$ for 5 and $10 \mathrm{~s}$. The textured samples were sonicated in distilled water, ethanol, and acetone for $10 \mathrm{~min}$ each. The cerium coatings were then deposited on the samples by immersion in two different solutions, which are discussed below.

Deposition in a Solution of Hexa-Hydrated Cerium Nitrate

Inside a vessel, we mixed the same amount of two aqueous solutions of hexahydrated cerium nitrate $\left(\mathrm{Ce}\left(\mathrm{NO}_{3}\right)_{3}-6 \mathrm{H}_{2} \mathrm{O}\right)$ (Sigma-Aldrich, St. Louis, MI, YSA) $0.05 \mathrm{M}$ and hexaethyltetramine $\left(\mathrm{C}_{6} \mathrm{H}_{12} \mathrm{~N}_{4}\right)$ (Sigma-Aldrich, St. Louis, MI, YSA) $0.5 \mathrm{M}$. The mixture was heated at $70{ }^{\circ} \mathrm{C}$ and then the samples

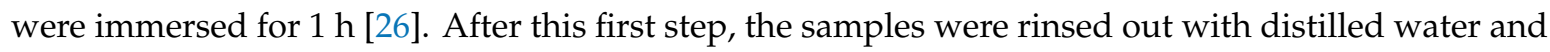
dried with a jet of hot air. To ensure a total removal of solvent traces, the samples were again dried in an oven at $80^{\circ} \mathrm{C}$ for $1 \mathrm{~h}$. Subsequently, the samples were immersed for $2 \mathrm{~h}$ at room temperature in a solution of stearic acid/ethanol at $1 \%$ in weight (both chemicals were supplied by Sigma-Aldrich). The samples were rinsed out with ethanol and dried with a jet of hot air. Finally, the samples were dried in an oven at $60^{\circ} \mathrm{C}$ for $2 \mathrm{~h}$ (see scheme of Figure 2).

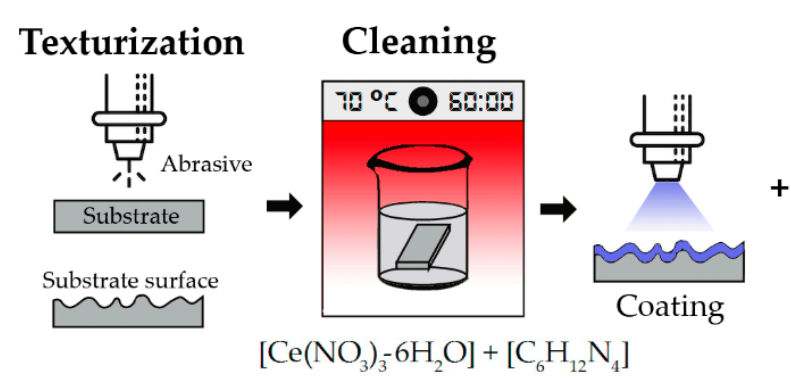

\section{Hydrophobization}

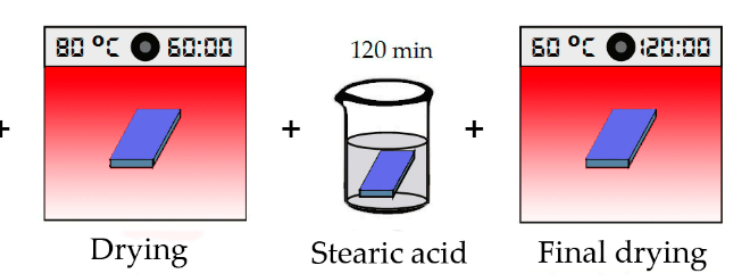

Figure 2. Scheme for the deposition of cerium-based coatings by immersion using cerium nitrate.

Deposition in a Solution of Hepta-Hydrated Cerium Chloride

Following the above procedure, the textured and cleaned samples were immersed into a vessel containing a mixture of $50 \mathrm{~mL}$ distilled water, $2 \mathrm{~g}$ heptahydrated cerium (III) chloride $\left(\mathrm{CeCl}_{3}-7 \mathrm{H}_{2} \mathrm{O}\right)$, and $3 \mathrm{~mL}$ hydrogen peroxide $\left(\mathrm{H}_{2} \mathrm{O}_{2}\right)$ (Sigma-Aldrich Química SL, Madrid, Spain) at $40{ }^{\circ} \mathrm{C}$ for $1 \mathrm{~h}$ [30]. After this time, the samples were introduced into a second vessel containing a $0.03 \mathrm{M}$ solution of stearic 
acid in ethanol for $10 \mathrm{~min}$ at room temperature (see scheme of Figure 3). Finally, the samples were removed from the solution, cleaned, and dried following the same strategy as in the previous case without the intermediate drying stage.

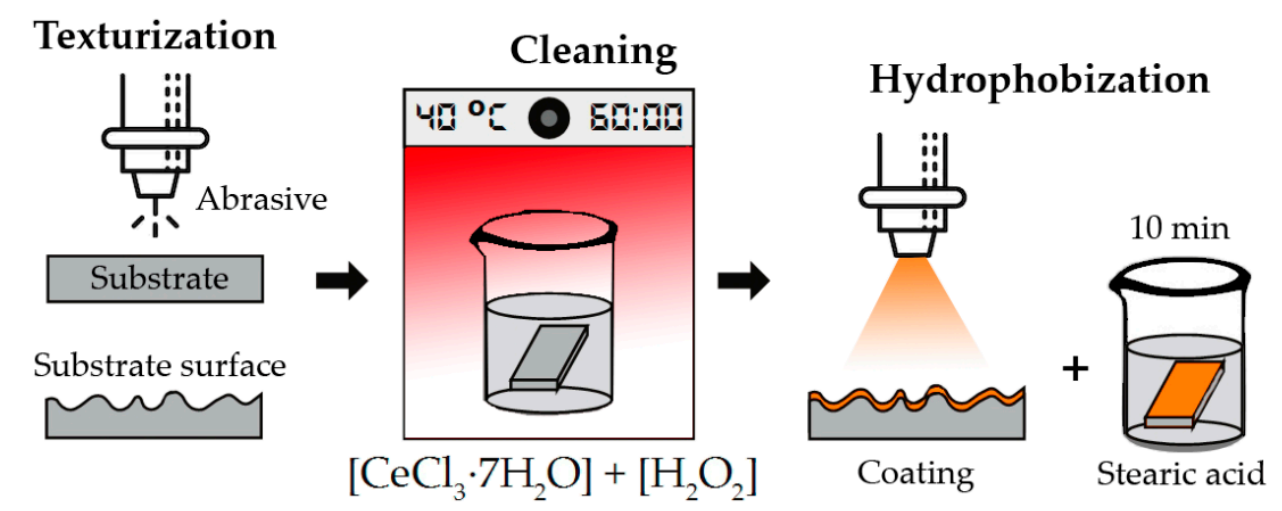

Figure 3. Scheme for the deposition of cerium-based coatings by immersion using cerium chloride.

\subsection{Wettability Analysis}

The wettability of the surfaces was analyzed by means of the tilting plate and bouncing drop methods. The first of these provides the advancing contact angle (ACA), receding contact angle (RCA), and sliding angle (SA) of water drops. These parameters may reveal the water shear adhesion of the surface. The bouncing drop method provides the number of bounces given by a water drop on a water-repellent surface, which is connected to its water tensile adhesion.

Tilting plate experiments were carried out with a tilting apparatus, the details of which having been given elsewhere [29]. For each experiment, a $50 \mu \mathrm{L}$ drop of Milli-Q water was deposited on the sample. The sample was fixed to a platform which was initially in a horizontal position and then was inclined at $5^{\circ} / \mathrm{s}$. During the inclination process, several side-view images of the drop were captured with a camera attached to the platform. At increasing tilting angles, the drop was deformed (loss of axisymmetric shape) and the downhill contact angle increased while the uphill contact angle decreased. At a certain tilt angle, the drop initiated a global motion, which means that the entire contact line was displaced on the surface. This critical tilt angle is known as the sliding angle (SA). For superhydrophobic surfaces, this angle may be referred to as the roll-off angle and it is not greater than $10^{\circ}$. Before and after the incipient sliding of the inclined drop, ACA and RCA are determined at both sides of the drop profile by simple goniometry. The reported ACA, RCA, and SA values of each sample correspond to the values averaged over at least three runs (three different drops placed at different positions on the sample).

The bouncing drop experiments were only conducted for superhydrophobic surfaces. These experiments are useful to distinguish the degree of superhydrophobicity of low-adhesion surfaces. These experiments consist of counting the number of bounces given by a drop that is released from a certain height from the surface and impacts over it. After the impact, if the surface is water-repellent, the drop bounces. The lower the tensile adhesion of the surface the lower damping induced by the surface during the impact, and, therefore, the higher number of bounces. A microliter-syringe (needle of $0.25 \pm 0.05 \mathrm{~mm}$ diameter) was filled with Milli-Q water and fixed vertically on the surface. The distance between the tip of the needle and the surface was fixed to $24.00 \pm 0.05 \mathrm{~mm}$. The syringe plunger was moved gently by means of a micrometer screw to avoid vibrations. When the drop reaches a certain limit volume, it falls down by gravity. Typically, the limit volume was $4.2 \mu \mathrm{L}$. The drop impacts on the surface and it bounces. We counted the number of bounces until the drop rested on the surface. The entire sequence was recorded with a Phantom Miro 4 high speed monochromatic camera (Iberoptics S.L.U, Madrid, Spain). This test was repeated at least three times for each substrate. We report here the average number of bounces. 


\subsection{Roughness and Surface Morphology Analysis}

To measure the surface roughness, we used a contact profilometer Mitutoyo SJ-201 (Grupo Unceta, Guipúzcoa, Spain) over a scanning length of $2.5 \mathrm{~mm}$. We have reported the average roughness values (Ra). The SEM images and EDX analysis were conducted with a JEOL JSM 7800F Prime high-resolution scanning electron microscope (JEOL USA, Inc., Peabody, MA, USA). To explore the surface features at the micro scale and nano scale, we set the scanning areas as $(100 \times 100) \mu \mathrm{m}^{2}$ and $(1 \times 1) \mu \mathrm{m}^{2}$ with different magnifications.

\section{Results and Discussion}

\subsection{Cathodic-Electrodeposited Coatings}

Figure 4 shows the SA results in terms of the electric voltage applied for the AS and Al-Mg alloy samples at different deposition times. We observed that the most efficient treatment (minimum SA value $\sim 5^{\circ}$ ) for the $\mathrm{Al}-\mathrm{Mg}$ alloy corresponded to a deposition time of $30 \mathrm{~min}$ and a voltage of $40 \mathrm{~V}$. For the AS sample, we observed the highest efficiency $\left(\mathrm{SA}<5^{\circ}\right.$ ) for $10 \mathrm{~min}$ of deposition time at $30 \mathrm{~V}$ (see a typical pearl-shaped drop deposited on this sample in Figure $4 b$ ).

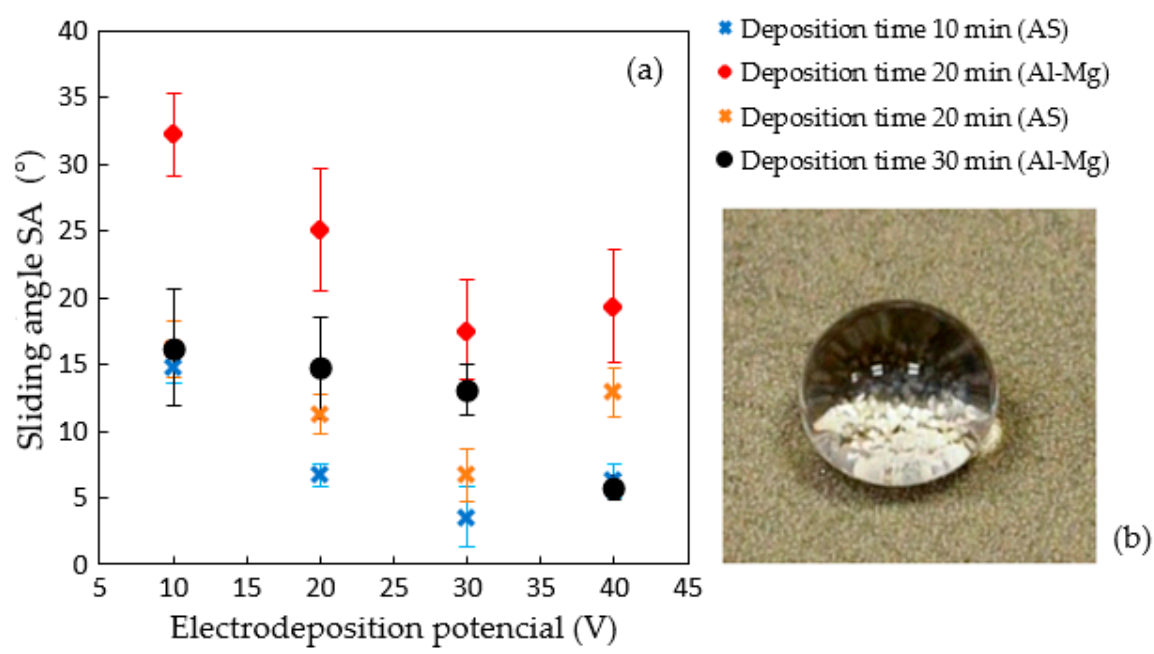

Figure 4. (a) Sliding angle (SA) of $50 \mu \mathrm{L}$ water drops on Al-Mg and AS substrates subjected to electrodeposition with cerium chloride and myristic acid at different voltages $\left(0.3 \mathrm{~mA} / \mathrm{cm}^{2}\right)$ for 10,20 , and $30 \mathrm{~min}$. (b) Image of water drop placed on a treated AS substrate (30 V and $10 \mathrm{~min}$ ).

In Table 2, we show the wettability and roughness results of the treated samples and the control (untreated) samples. Irrelevant results of the rest of the sandblasted AS samples are not reported here.

Table 2. Advancing contact angle (ACA), receding contact angle (RCA), SA, roughness (Ra), and number of bounces for the most representative samples after electrodeposition.

\begin{tabular}{cccccc}
\hline Substrate & Micro-Texturing & Time $(\mathbf{m i n}) /$ Voltage $(\mathbf{V})$ & Ra $(\mu \mathbf{m})$ & ACA/RCA/SA $\left(^{\circ}\right)$ & Bounces \\
\hline AS & - & - & $0.41 \pm 0.03$ & $122 / 91 / 29$ & - \\
AS & Sandpaper and & $10 / 30$ & $4.57 \pm 0.41$ & $155.5 / 142.3 / 3.6$ & 9 \\
Al-Mg & blasting at 0.3 MPa/10s & - & $0.23 \pm 0.03$ & $119 / 87 / 34$ & - \\
$\mathrm{Al}-\mathrm{Mg}$ & Sandpaper & $30 / 40$ & $2.41 \pm 0.32$ & $157.5 / 131.8 / 6.2$ & 6 \\
\hline
\end{tabular}

In Figure 4, except for the Al-Mg substrate (30 min), we found very similar trends of SA in terms of applied voltage. The minimum SA value was found when we applied a voltage of $30 \mathrm{~V}$. A similar trend has been reported earlier for copper surfaces. In that case, a minimum SA value was identified between 15 and $20 \mathrm{~V}$ [28]. However, we found differences in SA as a function of deposition time. For the Al-Mg 
substrates, we observed that longer depositions decreased the SA values. Instead, shorter depositions on the AS substrates led to lower SA values.

The lowest values of the sliding angle for the aluminized steel samples were reached for those samples whose Ra ranged between 4.5 to $5.5 \mu \mathrm{m}$, while the lowest values of the sliding angle for the Al-Mg samples were reached for those samples whose Ra values ranged between 2.5 and $3 \mu \mathrm{m}$ (see supplementary data). The roughness and wettability results for the most representative samples shown in Table 2 point out that the treated samples may be considered as water-repellent within the Cassie-Baxter regime. This is validated by the fact that both ACA and RCA increased with roughness.

In Figure 5, we show several SEM images of a selection of the samples compiled in Table 2. It may be observed that both surfaces are rough at the micro and nano scales. Since the Al-Mg alloy surfaces were polished but not sandblasted, their roughness at the micro scale can be seen to be lower than for the AS samples. This can be confirmed by comparing the roughness results displayed in Table 2. Figure $5 b$,d shows that the electrodeposition method creates a cauliflower-like structure due to the growth of cerium particles [28,29]. We found that the size of the nano-asperities formed on the AS samples were smaller than for the Al-Mg samples due to the greater number of preferential sites. In summary, the SEM images confirm the hierarchical structure of the treated samples which is responsible in part for their water-repellency properties.

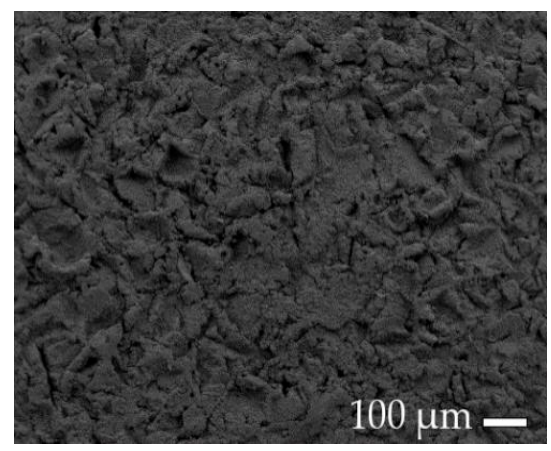

(a)

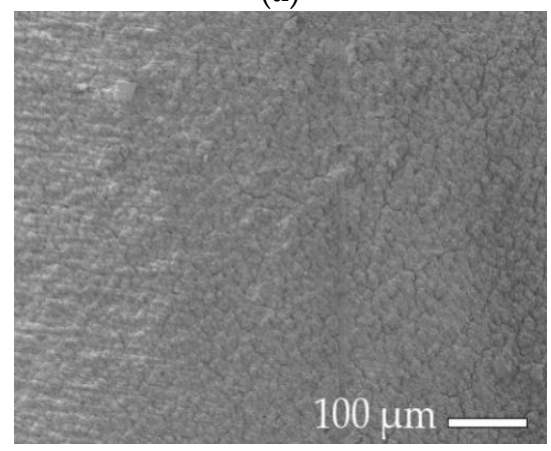

(c)

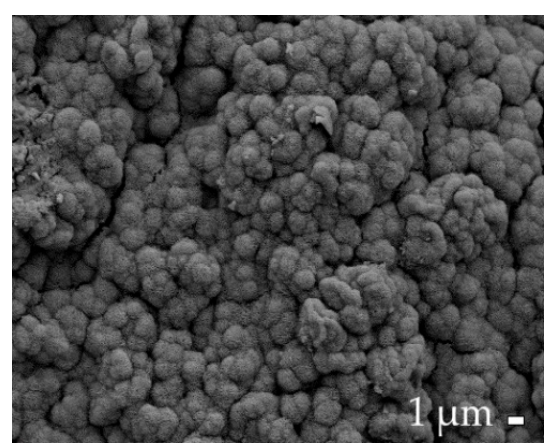

(b)

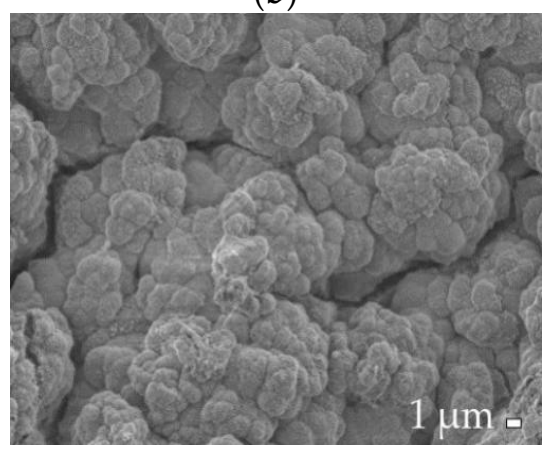

(d)

Figure 5. SEM images of the surface morphology of a textured AS sample at (a) magnification $\times 100$ and (b) $\times 4000$ after electrodeposition at $30 \mathrm{~V}$ for $10 \mathrm{~min}$, and a textured Al-Mg sample at (c) magnification $\times 180$ and $(\mathbf{d}) \times 3000$ after electrodeposition at $40 \mathrm{~V}$ for $30 \mathrm{~min}$.

Finally, in Figure 6 we show the EDX spectra obtained before and after the electrodeposition. In the electrodeposited samples (Figure $6 \mathrm{c}, \mathrm{d}$ ), the carbon signal is dominated by the presence of myristic acid on the uppermost coating (see Figure 1). In both cases, the presence of Ce with $18.1 \%$ and $20.1 \%$, respectively, and oxygen with $11.3 \%$ and $13.1 \%$ are also relevant, which indicates a proper degree of coverage of cerium after the electrodeposition method. 


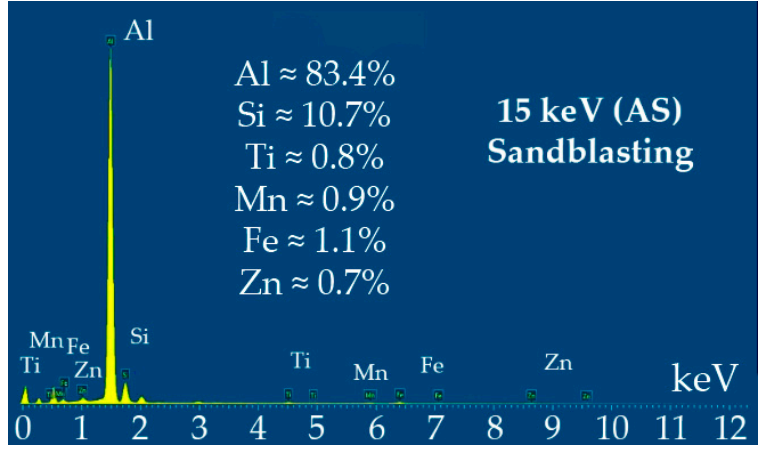

(a)

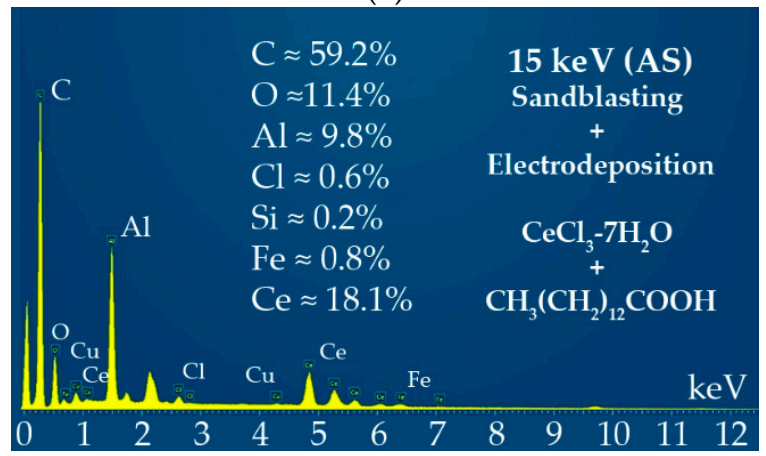

(c)

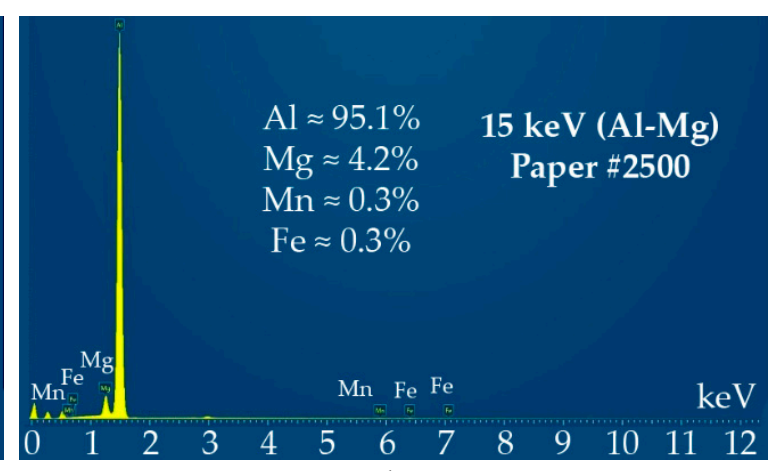

(b)

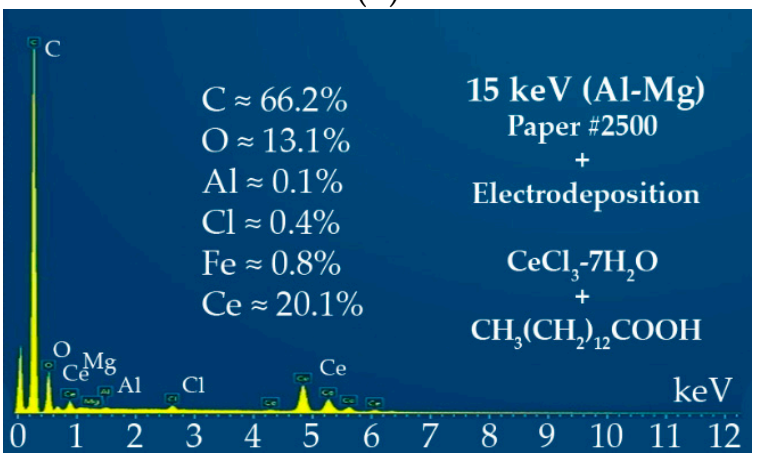

(d)

Figure 6. Energy-dispersive X-ray spectroscopy (EDX) spectra of (a) sandblasted AS substrate, (b) sanded $\mathrm{Al}-\mathrm{Mg}$ substrate, (c) treated AS substrate after electrodeposition (cerium chloride and myristic acid) at $30 \mathrm{~V}$ for $10 \mathrm{~min}$, and (d) treated $\mathrm{Al}-\mathrm{Mg}$ substrate after electrodeposition at $40 \mathrm{~V}$ for $30 \mathrm{~min}$.

\subsection{Immersion in Hexa-Hydrated Cerium Nitrate and Hepta-Hydrated Cerium Chloride}

Figure 7 shows the results for the AS and Al-Mg substrates after immersion in solutions of cerium nitrate salt (Figure 7a) and cerium chloride salt (Figure 7b) and later hydrophobization with stearic acid. Unlike for the electrodeposition method, the degree of micro-scale roughness of the substrates was crucial to reach superhydrophobicity. The sandblasting process was tuned in terms of blasting time and pressure. We selected in Table 3 the optimal results of Figure 7 following a balance between the lower SA values and lower surface damage caused by the previous sandblasting.
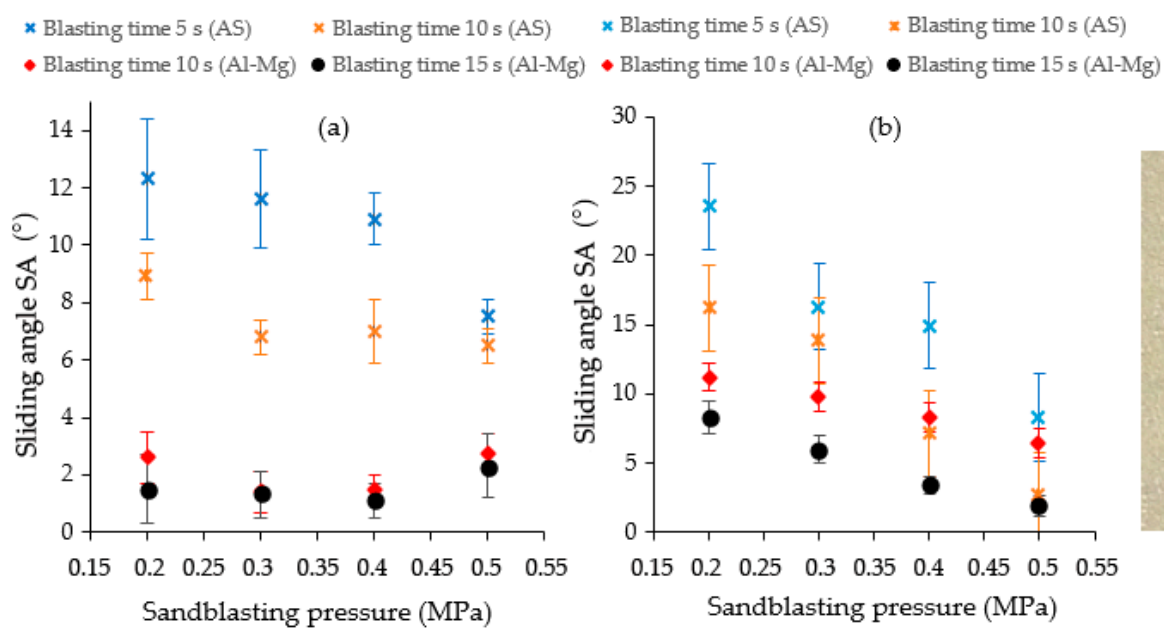

(c)

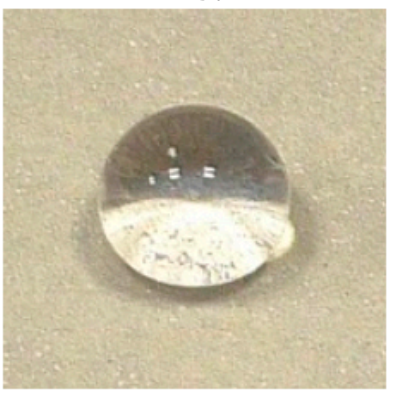

Figure 7. SA of $50 \mu \mathrm{L}$ water drops on $\mathrm{Al}-\mathrm{Mg}$ and AS substrates sandblasted at different pressures from 0.2 to $0.5 \mathrm{MPa}$ for 5,10 , and $15 \mathrm{~s}$ and immersed in solutions of (a) cerium nitrate and (b) cerium chloride. (c) Image of water drop placed on a treated Al-Mg substrate sandblasted at $0.4 \mathrm{MPa}$ for $15 \mathrm{~s}$. 
From Figure 7, one may conclude that regardless of the salt used, the longer blasting times, the higher the water repellency. However, we did not find a good agreement in the sandblasting pressures. In the case of cerium nitrate salt, although the pressure does not seem to play a crucial role, the optimal water repellency was reached when the pressure was between 0.3 and $0.4 \mathrm{MPa}$. However, for the cerium chloride salt, we found that the higher the pressure, the lower the sliding angle.

The morphology of the same samples compiled in Table 3 was analyzed by SEM and their results are shown in Figure 8. Again, these surfaces show a hierarchical texture as they are all rough at the micro scale (Figure 8, left) and nano scale (Figure 8, right). In this case, the nano-structure of the samples are clusters of particles with different degrees of adsorption and polydispersity. The size of these nano-asperities is substantially smaller than that observed for the electrodeposited samples (Figure 5, right). This might explain the better water-repellency results. In addition, the distribution of nano-asperities is less homogeneous for the sample shown in Figure 8b, which explains its worse wetting performance compared to the rest of the samples prepared by immersion. Figure $8 \mathrm{e}-\mathrm{h}$ shows typical accumulations and cluster dispositions similar to superhydrophobic textures of some cases studied with $\mathrm{Mg}$ alloys [31] and aluminium alloys of the 7000 series [32].

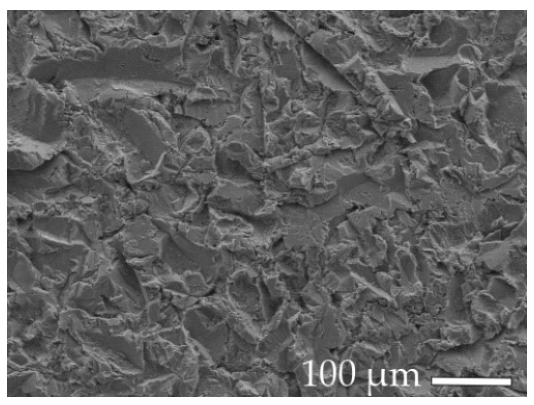

(a)

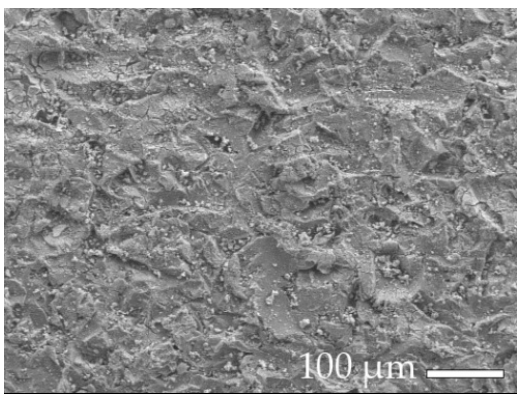

(c)

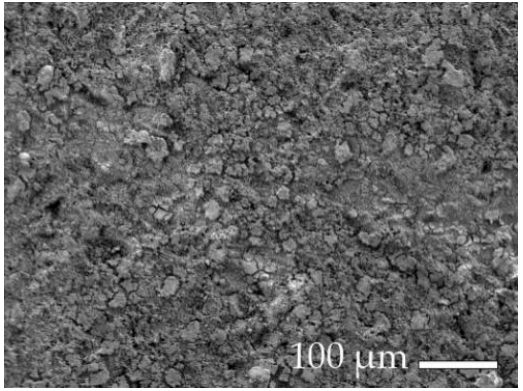

(e)

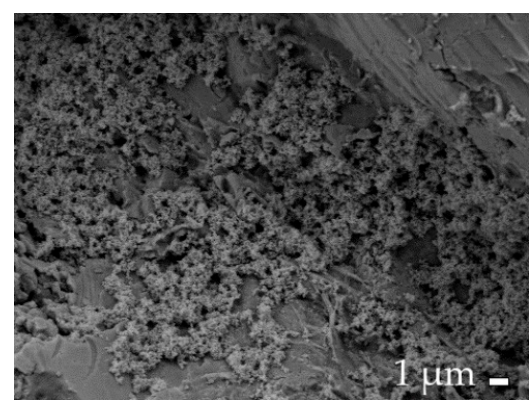

(b)

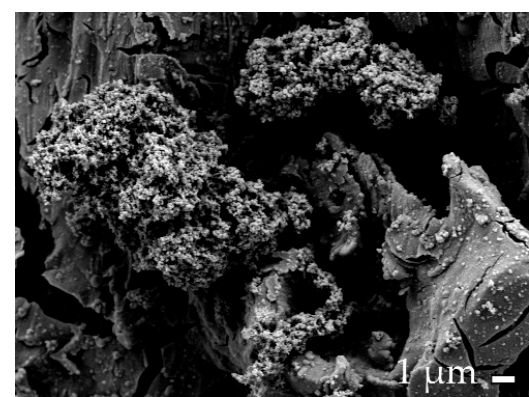

(d)

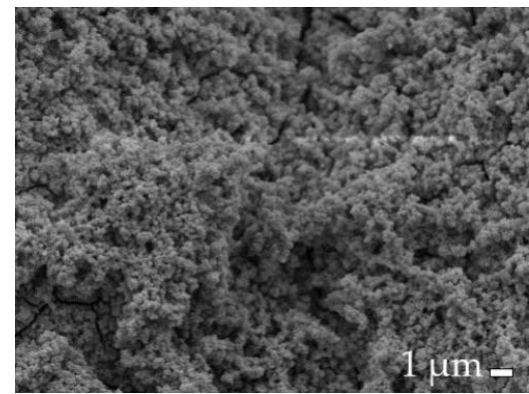

(f)

Figure 8. Cont. 


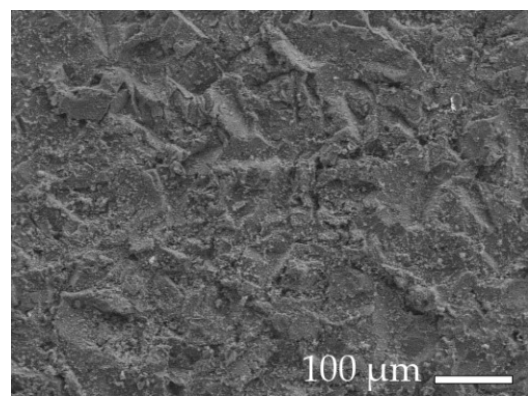

(g)

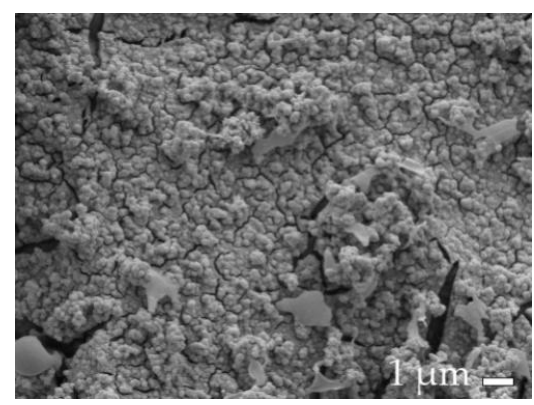

(h)

Figure 8. SEM images of the surface textures obtained using the immersion method: (a) AS sandblasted at $0.5 \mathrm{MPa}$ for $10 \mathrm{~s}$, (b) AS after immersion in cerium nitrate and stearic acid, (c) AS sandblasted at $0.5 \mathrm{MPa}$ for $10 \mathrm{~s}$, (d) AS after immersion in cerium chloride and stearic acid, (e) Al-Mg sandblasted at $0.4 \mathrm{MPa}$ for $10 \mathrm{~s}$ (f) after immersion in cerium nitrate and stearic acid, and (g) Al-Mg sandblasted at $0.5 \mathrm{MPa}$ for $15 \mathrm{~s}$ and $(\mathbf{h})$ after immersion in cerium chloride and stearic acid.

We also studied the elemental chemical composition by EDX for the same substrates analyzed in Figure 9, and the obtained spectra are shown in Figure 8. Unlike for the electrodeposited samples, the EDX spectra for all the immersed samples reveal a high signal for aluminium. This points to a thin or heterogenous coating. This issue is more noticeable for the AS samples (Figure 9a,b) because the presence of $\mathrm{Al}$ exceeds $40 \%$. This might explain the worse results obtained for this substrate in comparison with the Al-Mg alloy. In the case of the Al-Mg alloy samples (see Figure 9c,d), the cerium signal is dominant, and this points to a more homogeneous coating. For the rest of the elements detected, their concentrations are compatible with the reagents used in the protocol.

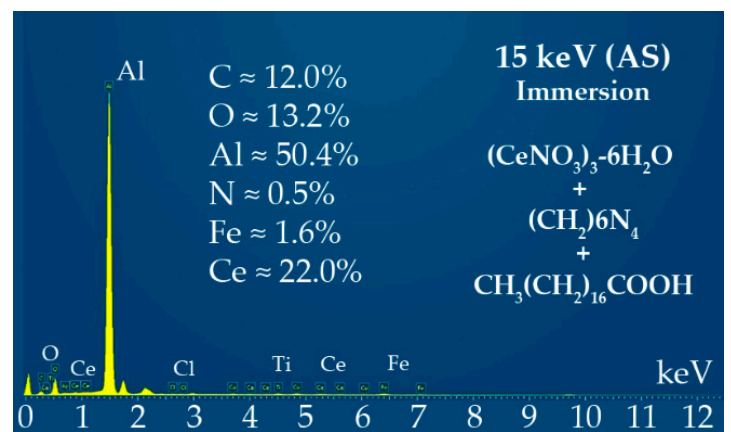

(a)

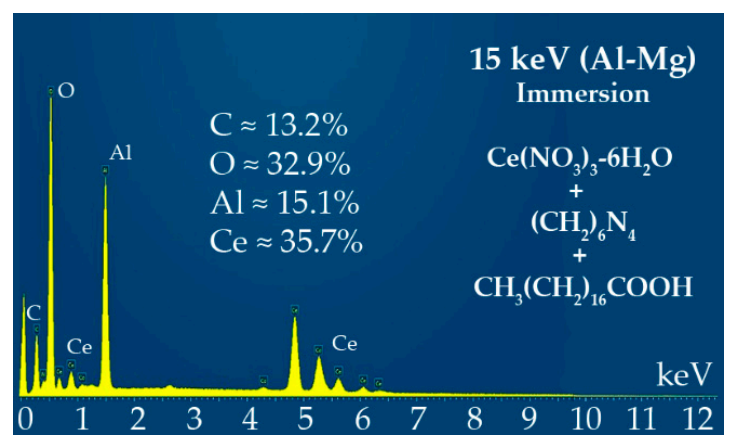

(c)

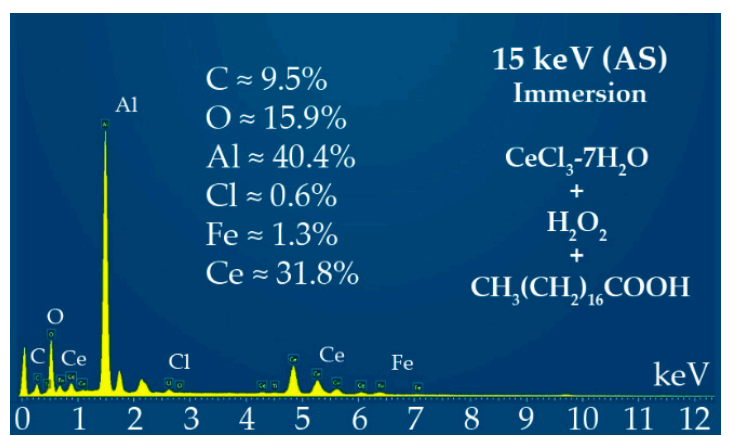

(b)

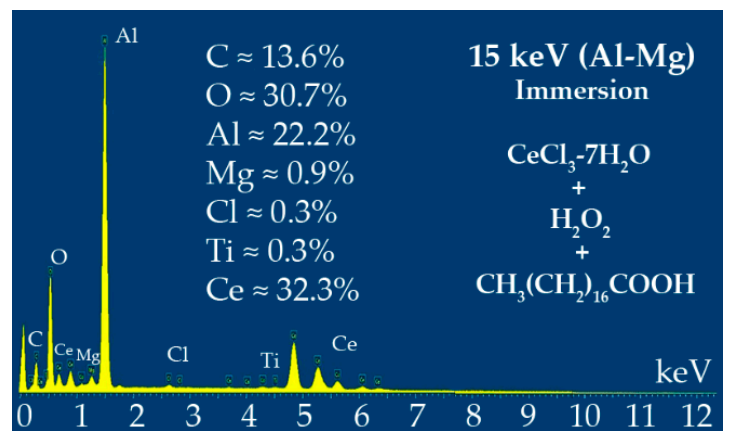

(d)

Figure 9. EDX spectra of (a) AS after immersion in cerium nitrate and stearic acid, (b) AS after immersion in cerium chloride and stearic acid, (c) Al-Mg after immersion in cerium nitrate and stearic acid, and (d) Al-Mg after immersion in cerium chloride and stearic acid. 
The samples and surface treatments indicated in Table 3 were used to perform the bouncing drop experiments and the results are also included in the last column of this table. In this case, we found a very good degree of superhydrophobicity for the Al-Mg samples, regardless of the salt used. However, for the AS samples we found that immersion in cerium chloride seemed to be more efficient than with cerium nitrate. These results agree reasonably well with the ones reported in Figure 7.

Table 3. Parameters for the most efficient samples used in this study: sandblasting settings, salt used in the immersion, ACA, RCA, SA, average Ra, and number of bounces.

\begin{tabular}{cccccc}
\hline Substrate & Blasting (MPa)/Time (s) & Salt & Ra $(\mu \mathrm{m})$ & ACA/RCA/SA $\left({ }^{\circ}\right)$ & Bounces \\
\hline AS & $0.5 / 10$ & Cerium nitrate & $3.37 \pm 0.33$ & $142.7 / 129.3 / 6.5$ & 8 \\
AS & $0.5 / 10$ & Cerium chloride & $4.03 \pm 0.74$ & $150.9 / 137.3 / 2.7$ & 14 \\
Al-Mg & $0.4 / 10$ & Cerium nitrate & $4.45 \pm 0.27$ & $155.5 / 138.3 / 1.5$ & 16 \\
Al-Mg & $0.5 / 15$ & Cerium chloride & $3.69 \pm 0.61$ & $155.1 / 136.3 / 2.3$ & 15 \\
\hline
\end{tabular}

About the relationship between roughness and wetting properties for the aluminized steel samples, we found the minimum sliding angle values for those samples with Ra values ranged between 3.0-3.5 $\mu \mathrm{m}$ when using nitrate chloride salt within the protocol. When cerium chloride was used, the Ra values leading to the lowest sliding angles ranged between $4.0 \mu \mathrm{m}$ and $4.5 \mu \mathrm{m}$. In the Al-Mg alloy samples, the optimization was reached for Ra values ranging 3.5-4.5 $\mu \mathrm{m}$ regardless of the salt used.

\section{Conclusions}

Several routes for producing superhydrophobic AS and Al-Mg surfaces have been proposed in this paper. The reduction of adhesion properties (low wetting properties) has been addressed through the incorporation of hierarchical structures by a two-step process based on sandblasting and subsequent cerium deposition using two types of cerium salts (cerium chloride and cerium nitrate) and two different strategies: (i) cathodic electrodeposition and (ii) adsorption by aqueous immersion. For the Al-Mg substrates, our results show that the most efficient protocol is based on immersion in cerium nitrate solution with previous sandblasting ( $0.4 \mathrm{MPa}$ for $10 \mathrm{~s})$. The electrodeposition method was less efficient athough it was also less invasive. For the AS substrates, we found that both the electrodeposition and immersion methods revealed a similar performance. With electrodeposition, an optimal scenario was obtained with the use of cerium chloride, an applied voltage of $30 \mathrm{~V}$, and a deposition time of $10 \mathrm{~min}$. With immersion, the best performance was observed with cerium chloride and previous sandblasting $(0.5 \mathrm{MPa}$ for $10 \mathrm{~s})$. The strategies developed using these multi-stage procedures allow for the obtaining of more functional and durable coatings. In this particular case, the incorporation of cerium produces a superhydrophobic product which is resistant to corrosion coating. Although some durability tests of the created samples were conducted, we found that the strategies used here did not result in durable coatings. Further work should be done to substantially improve the coating-substrate join and to quantify the mechanical durability.

Supplementary Materials: The following are available online at http://www.mdpi.com/2079-6412/9/12/774/s1.

Author Contributions: Conceptualization, G.G.-V., M.A.R.-V., and F.J.M.R.-C.; methodology, Ó.R.-A., P.C.-M., F.A.-S., and G.G.-V.; validation, G.G.-V., M.A.R.-V., and F.J.M.R.-C.; formal analysis, E.M.-R. and P.E.R.; investigation, P.C.-M. and F.A.-S.; resources, G.G.-V. and M.A.R.-V.; data curation, G.G.-V.; writing-original draft preparation, G.G.-V., F.J.M.R.-C., and Ó.R.-A.; writing—review and editing, all authors; visualization, all authors; supervision, G.G.-V., M.A.R.-V., and F.J.M.R.-C.; project administration, G.G.-V.; funding acquisition, M.A.R.-V. and P.E.R.

Funding: The authors were supported by the University of Cordoba (Spain), which financed this work through the Own Research Plan 2019. This research was partially financed by the State Research Agency (SRA) and European Regional Development Fund (ERDF) through the project MAT2017-82182-R.

Acknowledgments: The authors thank TECNIMACOR S.L (Córdoba, Spain) for their supply of the different substrates studied and practical information for the development of the research. In the same way, we would like to thank Professor Francisco Moyano, honorary collaborator of the University of Córdoba (Spain), for his thoughtful and useful advice. 
Conflicts of Interest: The authors declare no conflict of interest.

\section{References}

1. Rodríguez-Alabanda, Ó.; Romero, P.; Guerrero-Vaca, G. Evaluation of substrates of al-mg and aluminized steel coated with non-stick fluoropolymers after the removal of the coating. Materials 2018, 11, 2309. [CrossRef] [PubMed]

2. Rossi, S.; Gai, G.; Benedetto, R. De Functional and perceptive aspects of non-stick coatings for cookware. Mater. Des. 2014, 53, 782-790. [CrossRef]

3. Sánchez-Urbano, F.; Paz-Gómez, G.; Rodríguez-Alabanda, O.; Romero, P.E.; Cabrerizo-Vílchez, M.; Rodríguez-Valverde, M.A.; Guerrero-Vaca, G. Non-Stick coatings in aluminium molds for the production of polyurethane foam. Coatings 2018, 8, 301. [CrossRef]

4. Thomas, P. The use of fluoropolymers for non-stick cooking utensils. Surf. Coat. Int. 1998, 81, 604-609. [CrossRef]

5. Sethi, S.K.; Manik, G. Recent progress in super hydrophobic/hydrophilic self-cleaning surfaces for various industrial applications: A review. Polym. Plast. Technol. Eng. 2018, 57, 1932-1952. [CrossRef]

6. Piscitelli, F.; Tescione, F.; Mazzola, L.; Bruno, G.; Lavorgna, M. On a simplified method to produce hydrophobic coatings for aeronautical applications. Appl. Surf. Sci. 2018. [CrossRef]

7. Ganesh, V.A.; Raut, H.K.; Nair, A.S.; Ramakrishna, S. A review on self-cleaning coatings. J. Mate. Chem. 2011, 21, 16304-16322. [CrossRef]

8. Ba, Z.; Dong, Q.; Zhang, X.; Qiang, X.; Cai, Z.; Luo, X. Cerium-based modification treatment of Mg-Al hydrotalcite film on AZ91D Mg alloy assisted with alternating electric field. J. Alloy. Comp. 2017, 695, 106-113. [CrossRef]

9. Ferrari, M.; Benedetti, A. Superhydrophobic surfaces for applications in seawater. Adv. Colloid Interf. Sci. 2015, 222, 291-304. [CrossRef]

10. Yang, Y.F.; Li, Y.; Li, Q.L.; Wan, L.S.; Xu, Z.K. Surface hydrophilization of microporous polypropylene membrane by grafting zwitterionic polymer for anti-biofouling. J. Membr. Sci. 2010, 362, 255-264. [CrossRef]

11. Hung, L.H.; Lin, R.; Lee, A.P. Rapid microfabrication of solvent-resistant biocompatible microfluidic devices. Lab Chip 2008, 8, 983. [CrossRef] [PubMed]

12. Manca, M.; Cannavale, A.; De Marco, L.; Aricò, A.S.; Cingolani, R.; Gigli, G. Durable superhydrophobic and antireflective surfaces by trimethylsilanized silica nanoparticles-based sol-gel processing. Langmuir 2009, 25, 6357-6362. [CrossRef] [PubMed]

13. Tomšič, B.; Simončič, B.; Orel, B.; Černe, L.; Tavčer, P.F.; Zorko, M.; Jerman, I.; Vilčnik, A.; Kovač, J. Sol-gel coating of cellulose fibres with antimicrobial and repellent properties. J. Sol Gel Sci. Technol. 2008, 47, 44-57. [CrossRef]

14. Zimmermann, J.; Reifler, F.A.; Fortunato, G.; Gerhardt, L.C.; Seeger, S. A Simple, One-Step approach to durable and robust superhydrophobic textiles. Adv. Funct. Mater. 2008, 18, 3662-3669. [CrossRef]

15. Lin, D.; Zeng, X.; Li, H.; Lai, X.; Wu, T. One-Pot fabrication of superhydrophobic and flame-retardant coatings on cotton fabrics via sol-gel reaction. J. Colloid Interf. Sci. 2019, 533, 198-206. [CrossRef]

16. Xu, B.; Cai, Z. Fabrication of a superhydrophobic ZnO nanorod array film on cotton fabrics via a wet chemical route and hydrophobic modification. Appl. Surf. Sci. 2008, 254, 5899-5904. [CrossRef]

17. Guillermo, P.-Z.; Juan Carlos del, C.O.; Oscar, R.-A.; Pablo, E.-R.; Miguel, C.-V.; Guillermo, G.-V.; Miguel Angel, R.-V. Water-Repellent Fluoropolymer-Based Coatings. Coatings 2019, 9, 293. [CrossRef]

18. Karunakaran, R.G.; Lu, C.H.; Zhang, Z.; Yang, S. Highly transparent superhydrophobic surfaces from the coassembly of nanoparticles ( $\leq 100 \mathrm{~nm}$ ). Langmuir 2011, 27, 4594-4602. [CrossRef]

19. Cai, Y.; Chang, W.; Luo, X.; Qin, Y. Hydrophobicity of pyramid structures fabricated by micro milling. In Proceedings of the 2017 World Congress on Micro and Nano Manufacturing, Taiwan, China, 27 March 2017.

20. Ming, W.; Wu, D.; van Benthem, R.; de With, G. Superhydrophobic Films from Raspberry-like Particles. Nano Lett. 2005, 5, 2298-2301. [CrossRef]

21. Liu, Q.; Chen, D.; Kang, Z. One-Step electrodeposition process to fabricate corrosion-resistant superhydrophobic surface on magnesium alloy. ACS Appl. Mater. Interf. 2015, 7, 1859-1867. [CrossRef]

22. Qin, D.; Xia, Y.; Whitesides, G.M. Soft lithography for micro- and nanoscale patterning. Nat. Protoc. 2010, 5, 491-502. [CrossRef] [PubMed] 
23. Ruiz-Cabello, F.J.M.; Rodríguez-Criado, J.C.; Cabrerizo-Vílchez, M.; Rodríguez-Valverde, M.A.; Guerrero-Vacas, G. Towards super-nonstick aluminized steel surfaces. Prog. Org. Coat. 2017, 109, 135-143. [CrossRef]

24. Nouri, N.M.; Saadat-Bakhsh, M. Fabrication method of large-scale and mechanically durable superhydrophobic silicon rubber/aerogel coating on fibrous substrates. J. Coat. Technol. Res. 2017, 14, 477-488. [CrossRef]

25. Liu, C.; Su, F.; Liang, J.; Huang, P. Facile fabrication of superhydrophobic cerium coating with micro-nano flower-like structure and excellent corrosion resistance. Surf. Coat. Technol. 2014, 258, 580-586. [CrossRef]

26. Liang, J.; Hu, Y.; Fan, Y.; Chen, H. Formation of superhydrophobic cerium oxide surfaces on aluminum substrate and its corrosion resistance properties. Surf. Interf. Anal. 2013, 45, 1211-1216. [CrossRef]

27. Kaphle, A.; Navya, P.N.; Umapathi, A.; Daima, H.K. Nanomaterials for agriculture, food and environment: Applications, toxicity and regulation. Environ. Chem. Lett. 2018, 16, 43-58. [CrossRef]

28. Chen, Z.; Li, F.; Hao, L.; Chen, A.; Kong, Y. One-step electrodeposition process to fabricate cathodic superhydrophobic surface. Appl. Surf. Sci. 2011, 258, 1395-1398. [CrossRef]

29. Pedraza, F.; Mahadik, S.A.; Bouchaud, B. Synthesis of ceria based superhydrophobic coating on $\mathrm{Ni20 \textrm {Cr }}$ substrate via cathodic electrodeposition. Phys. Chem. Chem. Phys. 2015, 17, 31750-31757. [CrossRef]

30. Wan, B.; Ou, J.; Lv, D.; Xue, M.; Wang, F.; Wu, H. Superhydrophobic ceria on aluminum and its corrosion resistance. Surf. Interf. Anal. 2016, 48, 173-178. [CrossRef]

31. Liu, Q.; Kang, Z. One-step electrodeposition process to fabricate superhydrophobic surface with improved anticorrosion property on magnesium alloy. Mater. Lett. 2014, 137, 210-213. [CrossRef]

32. Rivera, B.F.; Johnson, B.Y.; O'Keefe, M.J.; Fahrenholtz, W.G. Deposition and characterization of cerium oxide conversion coatings on aluminum alloy 7075-T6. Surf. Coat. Technol. 2004, 176, 349-356. [CrossRef]

(C) 2019 by the authors. Licensee MDPI, Basel, Switzerland. This article is an open access article distributed under the terms and conditions of the Creative Commons Attribution (CC BY) license (http://creativecommons.org/licenses/by/4.0/). 